\title{
PTU-090 QUANTIFICATION OF MACROPHAGE MIGRATION INHIBITORY FACTOR MRNA EXPRESSION IN GASTRIC CANCER TISSUES AND ITS CLINICAL SIGNIFICANCE
}

doi:10.1136/gut.2011.239301.218

YMohri, ${ }^{*}$ K Tanaka, ${ }^{1}$ M Ohi, ${ }^{1}$ C Miki, ${ }^{1}$ T Mohri, ${ }^{2}$ M Kusunoki, ${ }^{1}$ PJ Johnson ${ }^{3}$ 'Department of Gastrointestinal Surgery, MIE University Graduate School of Medicine; ${ }^{2}$ Department of Surgery, Toyama Hospital, Tsu Mie, Japan; ${ }^{3}$ School of Cancer Sciences, University of Birmingham, Birmingham, UK

Introduction Macrophage migration inhibitory factor (MIF) is a proinflammatory cytokine derived from $\mathrm{T}$ cells and the pituitary gland. However, several types of solid cancers also secrete MIF, and this factor has been suggested to play an important role in carcinogenesis and the progression of malignancy. In this study, we quantified MIF mRNA expression of gastric cancer tissues and examined its relationship with clinicopathological factors.

Methods MIF mRNAs of both tumour and normal tissues were quantified by a real-time monitoring reverse-transcription PCR in 133 patients with gastric cancer. The relationship between the grade of MIF expression and clinicopathological factors such as body weight loss, cell type, stage, and prognosis was examined to investigate the clinical significance of intratumoral expression of MIF.

Results The mean value of MIF mRNA in tumour tissues was 1.56 , whereas that of normal gastric tissue was $0.38(\mathrm{p}=0.0011)$. The amounts of MIF proteins revealed by ELISA correlated with those of the corresponding mRNAs. Patients with protein malnutrition showed a significantly higher expression of MIF mRNA than other subjects and higher expression of MIF mRNA was significantly associated with unfavourable prognosis $(\mathrm{p}=0.0197)$.

Conclusion The general intratumoral expression and close relation with cancer associated malnutrition suggested that MIF might contribute to tumorigenes.

Competing interests None.

Keywords gastric cancer, MIF. 